\title{
Proof of an Outer Membrane Target of the Efflux Inhibitor Phe-Arg- $\beta$-Naphthylamide from Random Mutagenesis
}

\author{
Sabine Schuster ${ }^{1, *}$, Jürgen A. Bohnert ${ }^{2}$, Martina Vavra ${ }^{1}$, John W. Rossen ${ }^{3}{ }^{\mathbb{D}}$ and \\ Winfried V. Kern ${ }^{1,4}$ \\ 1 Division of Infectious Diseases, Department of Medicine II, University Hospital and Medical Center, \\ 79106 Freiburg, Germany; martina.vavra@uniklinik-freiburg.de (M.V.); \\ winfried.kern@uniklinik-freiburg.de (W.V.K.) \\ 2 Institute of Medical Microbiology, Greifswald University Hospital, 17475 Greifswald, Germany; \\ juergen.bohnert@uni-greifswald.de \\ 3 Department of Medical Microbiology and Infection Prevention, University of Groningen, University Medical \\ Center Groningen, 9713 Groningen, The Netherlands; j.w.a.rossen@rug.nl \\ 4 Faculty of Medicine, Albert-Ludwigs-University, 79085 Freiburg, Germany \\ * Correspondence: sabine.schuster@uniklinik-freiburg.de; Tel.: +49-761-270-35990
}

Academic Editor: Seung Seo Lee

Received: 10 January 2019; Accepted: 25 January 2019; Published: 29 January 2019

\begin{abstract}
Phe-Arg- $\beta$-naphthylamide $(\mathrm{PA} \beta \mathrm{N})$ has been characterized as an efflux pump inhibitor (EPI) acting on the major multidrug resistance efflux transporters of Gram-negative bacteria, such as AcrB in Eschericha coli. In the present study, in vitro random mutagenesis was used to evolve resistance to the sensitizing activity of $\mathrm{PA} \beta \mathrm{N}$ with the aim of elucidating its mechanism of action. A strain was obtained that was phenotypically similar to a previously reported mutant from a serial selection approach that had no efflux-associated mutations. We could confirm that acrB mutations in the new mutant were unrelated to PA $\beta N$ resistance. The next-generation sequencing of the two mutants revealed loss-of-function mutations in lp $x M$. An engineered lp $x M$ knockout strain showed up to 16-fold decreased PA $\beta \mathrm{N}$ activity with large lipophilic drugs, while its efflux capacity, as well as the efficacy of other EPIs, remained unchanged. LpxM is responsible for the last acylation step in lipopolysaccharide (LPS) synthesis, and lpxM deficiency has been shown to result in penta-acylated instead of hexa-acylated lipid A. Modeling the two lipid A types revealed steric conformational changes due to underacylation. The findings provide evidence of a target site of PA $\beta N$ in the LPS layer, and prove membrane activity contributing to its drug-sensitizing potency.
\end{abstract}

Keywords: PA $\beta N$; efflux pump inhibitor; random mutagenesis; lp $x M(m s b B)$; penta-acylated lipid A; permeabilizer

\section{Introduction}

Rapidly increasing rates of multidrug resistance (MDR) in aerobic and obligate anaerobic bacterial pathogens accompanied by the stagnating development of new antibiotics is one of the major public health challenges today. Alternative treatment options are urgently needed, and this may include the use of adjuvants sensitizing bacteria to antimicrobial drugs. With respect to Gram-negative bacteria, which are characterized by intrinsic resistance mechanisms due to an outer membrane (OM) barrier and MDR efflux pumps, drug potentiating agents, such as permeabilizers [1] and efflux pump inhibitors (EPIs) [2], offer a promising approach. The latter should be able to target resistance nodulation cell division (RND)-type transporters, which constitute the major MDR efflux systems in Gram-negatives. Examples are AcrAB-TolC from Escherichia coli and MexAB-OprM from Pseudomonas aeruginosa, with AcrB 
and MexB being the pumping components of these complexes. Most RND-type efflux pumps are characterized by an extremely broad substrate spectrum [3], and their role in the MDR of Gram-negative pathogens has been well demonstrated [4]. Agents from several chemical substance classes have been described as inhibitors of RND-type transporters [5], but none of them, to our knowledge, has reached clinical applicability so far. However, studying the mechanism(s) of action of these model EPIs could provide prospects regarding the design of new molecules. Such a model EPI is Phe-Arg- $\beta$-naphthylamide $(\mathrm{PA} \beta \mathrm{N})$, which is a cationic dipeptide with a naphthyl moiety and one of the first compounds reported to inhibit RND-type efflux pumps [6]. Frequently, it has been used to evaluate the efflux phenotype of Gram-negatives [7,8] and the putative substrate nature of drugs regarding RND-type transport [9]. However, from the first report of the compound, there have been indications that PA $\beta N$ is not a pure EPI, and an additional mode of action by a membrane-permeabilizing activity was postulated [6,10-13]. Exploring the mode of action of PA $\beta N$ was additionally complicated by the fact that the EPI itself is effluxed by RND transporters [6,14]. So far, insights of how PA $\beta N$ works have predominantly come from functional studies assessing the impact on drug susceptibility and the uptake of compounds [6,10-12], from co-crystallization experiments with AcrB [15] and computational approaches $[16,17]$. Since the results predominantly suggested target sites of PA $\beta N$ in the distal substrate binding pocket of $\mathrm{AcrB}$, a competitive mechanism of action could be expected. However, pronounced effects of the EPI in particular with macrolides and rifamycins, which both reveal binding specificities in the proximal substrate binding pocket [18-20], still remained unexplained.

Recently, we reported another approach to elucidate the mechanism of action of model EPIs. By using in vitro random mutagenesis, which is also designated as directed evolution, we were able to identify target sites of 1-(1-napthyl-methyl)-piperazine (NMP) in AcrB [21]. Within the scope of that earlier study, we had obtained a mutant derived from a serial selection procedure (also referred to as in vivo mutagenesis) revealing resistance to the drug-sensitizing activity of PA $\beta N$ ("PA $\beta N$-resistance"), but without any efflux-associated mutations, and with unknown reason for the observed phenotype. Here, we describe the generation of another PA $\beta N$-resistant mutant and the proof of an efflux-unrelated target of $\mathrm{PA} \beta \mathrm{N}$.

\section{Results and Discussion}

\subsection{PABN-Resistant Mutants from Random Mutagenesis Revealed Loss-of-Function Mutations in LpxM}

Previously reported PA $\beta N$-resistant mutant $C 5 / 1 / 17$ had been derived from the sequential selection of E. coli 3-AG100 with increasing concentrations of clarithromycin (CLR) in the presence of PA $\beta N$ [21]. Another method that was used in the same study, an in vitro random mutagenesis procedure directly targeting $a c r B$ by an error-prone PCR method, had failed to generate resistance to the drug-sensitizing action of the EPI. The approach had been applied to the gene regions encoding the periplasmic domain of $A c r B$, which is responsible for substrate recognition in RND-type transporters [22]. Since it could not be excluded that PA $\beta N$ acts in an allosteric manner, we now extended in vitro random mutagenesis to the whole of AcrB. From this directed evolution approach, $4 \times 10^{5}$ mutants were obtained and subsequently selected using a CLR-PA $\beta N$ combination that inhibits the growth of the parental E. coli strain 3-AG100. Several macrolides including CLR are confirmed substrates of AcrB [23] with pronounced susceptibility to the action of PA $\beta N$ (Figure 1a, Table S1). PA $\beta \mathrm{N}$ was used at $25 \mathrm{mg} / \mathrm{L}$, which is a concentration that has been demonstrated to exhibit high sensitizing potency while staying below the intrinsic MIC (minimum inhibitory concentration) in $a c r B$-deficient E. coli mutants [24]. A single mutant, CP1, revealing stable resistance to the activity of $\mathrm{PA} \beta \mathrm{N}$ with several drugs was achieved from the directed evolution procedure. As already seen with mutant C5/1/17, synergism in CP1 was decreased up to 16-fold with macrolides, rifamycins, and novobiocin (Figure 1a, Table S1). Again, a significant decline in PA $\beta N$ efficacy was predominantly limited to large lipophilic drugs with MW (molecular weight) $>600$. In contrast, marginal or no 
decreases in PA $\beta N$ activity were detected with smaller and/or more hydrophilic agents (Figure 1a, Table S1).

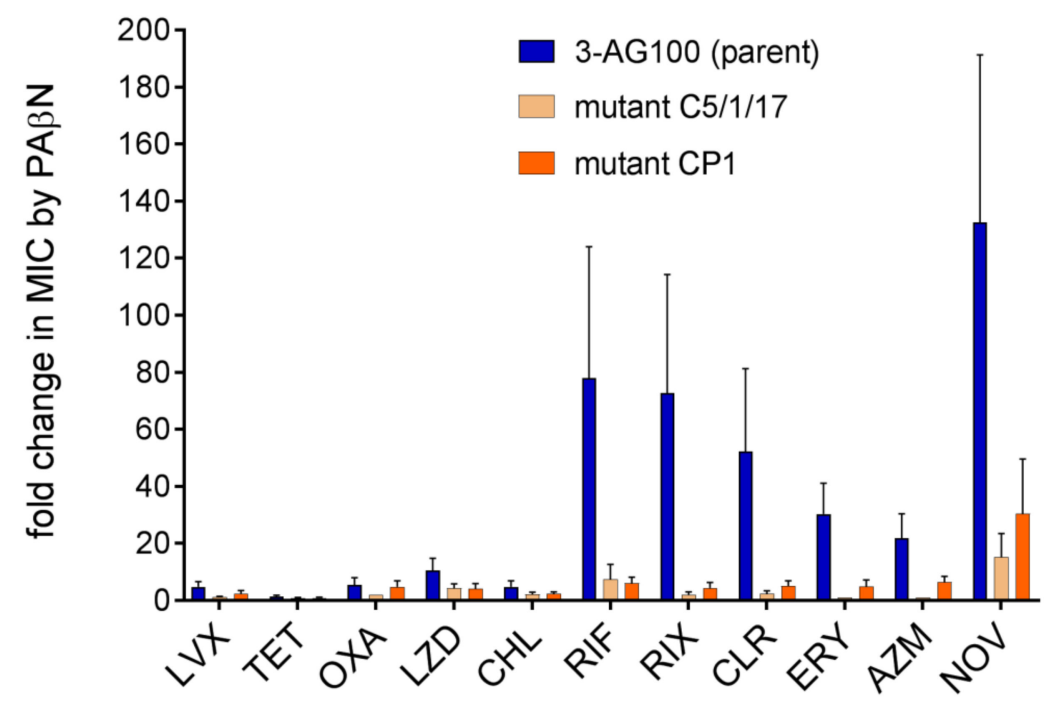

(a)

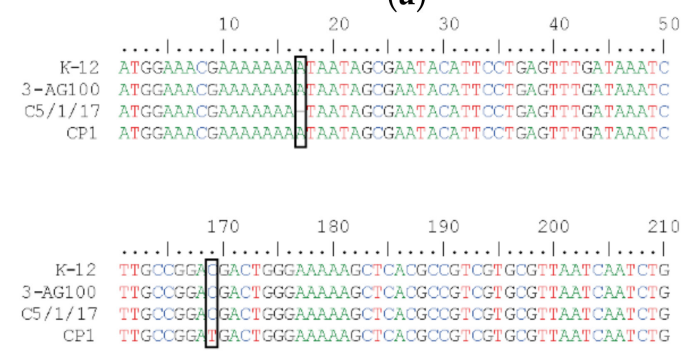

(b)

Figure 1. Phe-Arg- $\beta$-naphthylamide (PA $\beta N)$-resistant mutants versus parental E. coli 3-AG100 (a) PA $\beta N$ efficacies with drugs shown as ratios of minimum inhibitory concentrations (MICs) without and with $25 \mathrm{mg} / \mathrm{L}$ PA $\beta \mathrm{N}$; LVX, levofloxacin; TET, tetracycline; OXA, oxacillin; LZD, linezolid; CHL, chloramphenicol; RIF, rifampin; RIX, rifaximin; CLR, clarithromycin; ERY, erythromycin; AZM, azithromycin; NOV, novobiocin. Statistical significance determined for RIF, RIX, CLR, ERY, AZM, and NOV (mutants vs. parent 3-AG100), $p$-values $\leq 0.001(n=4)$; MICs are shown in Table S1. (b) Nucleotide region 1-50 and 161-210 of gene lpxM (reference sequence E. coli K-12, RefSeq NC_000913.3).

In contrast to mutant $C 5 / 1 / 17$ and as could be expected due to the error-prone PCR method applied, acrB from mutant CP1 harbored four single-nucleotide mutations encoding amino acid alterations V129I, L270V, T495S, and A873V. Surprisingly, their chromosomal reconstruction in parental strain 3-AG100 did not result in any PA $\beta N$-resistance (3-AG100acrB $B_{C P 1}$, Table S1). Next, we substituted mutated $a c r B$ in mutant $C P 1$ by wild-type $a c r B$, and found the PA $\beta N$ resistance phenotype maintained $\left(\mathrm{CP} 1\right.$ acrB $_{\mathrm{WT}}$, Table S1).

Even though directed evolution approaches were used, mutations outside the targeted gene region cannot be completely excluded. Thus, we also sequenced $a c r A$ and tolC encoding the accessory proteins of AcrB. However, as with PA $\beta N$-resistant mutant C5/1/17, no mutations were detected.

Consequently, next-generation sequencing (NGS) was performed. Whole genome variant analysis revealed only one alteration shared by both mutants, $\mathrm{C} 5 / 1 / 17$ and $\mathrm{CP} 1$, in comparison to their parent 3-AG100, which was the loss-of-function of $\operatorname{lp} x M(m s b B)$ by a frameshift and an early stop codon, respectively (Figure $1 b)$. L $p x M$ encodes an acyltransferase that is responsible for the last step in lipid A synthesis: the attachment of a secondarily bound myristic acid residue. Somerville et al. 
have demonstrated that the lipopolysaccharide (LPS) fatty acid pattern from lpxM-deficient E. coli mutants was lacking myristate, suggesting the occurrence of predominantly penta-acylated instead of hexa-acylated lipid A [25].

\subsection{Proved Impact on PAßN Efficacy from an LpxM Knockout Mutant}

To evaluate the impact of $\operatorname{lp} x M$ deficiency, we knocked out lpxM in parental strain 3-AG100. Similar to our findings with $C 5 / 1 / 17$ and $C P 1$, the $\Delta l p x M$ mutant revealed significantly decreased synergistic activity of $\mathrm{PA} \beta \mathrm{N}$ with large lipophilic drugs, whereas only marginal effects were seen with smaller and/or more hydrophilic compounds (Figure 2, Table S2). Since $\mathrm{Mg}^{2+}$ ions are known to contribute to OM integrity substantially [26], and it was speculated that they might be displaced by $\mathrm{PA} \beta \mathrm{N}$ due to its two positive charges under physiological conditions [6], we compared susceptibilities in LB (Luria/Miller broth) and in cation-adjusted medium containing higher and well-defined $\mathrm{Ca}^{2+}$ and $\mathrm{Mg}^{2+}$ concentrations. However, we could not detect any relevant impact of cation concentrations on changes in PA $\beta N$ efficacy (Tables S1 and S2). Nevertheless, to avoid effects due to unphysiologically low cation availability, further assays with the aim to characterize mutant $\Delta \mathrm{lpxM}$ were conducted in the presence of $\mathrm{mM}$ of $\mathrm{MgCl}_{2}$, and with cultures from cation-adjusted medium.
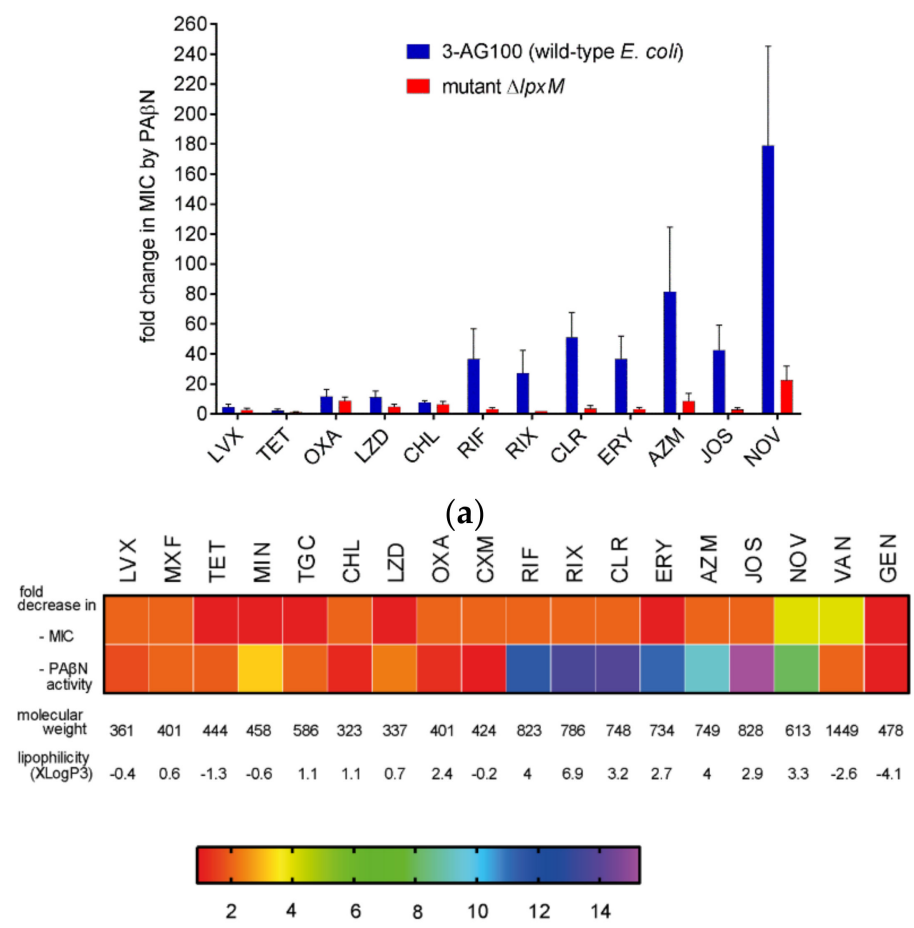

(b)

Figure 2. PA $\beta N$ activity in mutant $\triangle l p x M$ in comparison to its parent 3-AG100. (a) Ratios of MICs without and with $25 \mathrm{mg} / \mathrm{L}$ of PA $\beta \mathrm{N}$; Statistical significance ( $\triangle l p x M$ mutant vs. parent 3-AG100), $p$-values $<0.0001$ for RIF, RIX, CLR, ERY, AZM, and NOV; other $p$-values < 0.01, except for LVX ( $p$-value 0.02), and for OXA and CHL ( $p$-value $>0.1 ; n \geq 6)$. (b) Heat map depicting decreases in MIC $\left(\mathrm{MIC}_{3-\mathrm{AG} 100} / \mathrm{MIC}_{\Delta \mathrm{lpxM}}\right)$ and in PA $\beta \mathrm{N}$ activity $\left(\left(\mathrm{MIC}_{3-\mathrm{AG} 100} / \mathrm{MIC}_{3-\mathrm{AG} 100+\mathrm{PA} \beta \mathrm{N}}\right) /\left(\mathrm{MIC}_{\Delta \mathrm{lpxM}} / \mathrm{MIC}_{\Delta \mathrm{lpxM}+\mathrm{PA} \beta \mathrm{N}}\right)\right)$, color scale of the heat map represents $\mathrm{x}$-fold changes; molecular weights and computed partition coefficients $\mathrm{X} \log \mathrm{P} 3$ of drugs from PubChem [27]. LVX, levofloxacin; MXF moxifloxacin; TET, tetracycline; MIN, minocycline; TGC, tigecycline; CHL, chloramphenicol; LZD, linezolid; OXA, oxacillin; CXM, cefuroxime; RIF, rifampin; RIX, rifaximin; CLR, clarithromycin; ERY, erythromycin; AZM, azithromycin; JOS, josamycin; NOV, novobiocin; VAN, vancomycin; GEN, gentamicin. MICs are provided in Tables S1 and S2.

In contrast to small and rather hydrophilic drugs (considered to use predominantly porins to permeate into the bacterial cell), large and/or more hydrophobic compounds are thought to pass the 
OM bilayer by lipid-mediated pathways, meaning passive diffusion or self-promoted uptake [26,28]. It was also suggested that some agents, such as tetracyclines and quinolones, use both porin as well as lipid-mediated pathways [28]. This could explain the different effects on the PA $\beta N$ activity that were detected with several of these antibiotics (Figure 2, Table S2).

$\mathrm{PA} \beta \mathrm{N}$ efficacy changes could simply be due to alterations in susceptibilities to the respective synergistic drug. However, no correlations could be found when comparing these parameters (Figure 2b). In accordance with earlier reports of lpxM-deficient E. coli mutants [25,29-33], the susceptibilities to most drugs were only marginally increased or unchanged with few exceptions. The fourfold decreases in the MICs of novobiocin and vancomycin (Figure 2b, Table S2) indicated a higher OM permeability for these compounds. This might rather enhance the effectiveness of PA $\beta N$, but it was significantly decreased with novobiocin. Notably, resistance to the more hydrophilic and extremely large vancomycin was neither reducible by PA $\beta \mathrm{N}$ in wild-type E. coli 3-AG100 nor in the more susceptible $\Delta$ lp $x M$ mutant (Figure 2b, Table S2).

\subsection{Hexa-Acylated Lipid A Structure from Wild-Type E. coli versus Penta-acylated from LpxM Mutants}

Upon our request, the group of Wonpil Im has added the penta-acylated structure as a variant from the hexa-acylated LPS of E. coli K-12 to the CHARMM-GUI LPS Modeler platform [34]. Major structural changes were manifested by a significantly increased distance between the two intramolecular phosphates (Figure 3), which are supposed to play a major role in cross-linking the LPS molecules via divalent cations $[10,26]$. However, as already mentioned, we found a negligible impact of cation concentrations on PA $\beta N$ efficacy changes in the lp $x M$ mutant. Furthermore, a study with E. coli mutants harboring dephosphorylated lipid A revealed maintained OM integrity despite decreased cationic stabilization options [35]. In contrast to the intramolecular lipid A phosphates, the remaining fatty acid chains have moved closer together in the penta-acylated lipid A structure (Figure 3). It could be speculated that their tighter package cause altered incorporation options between adjacent LPS molecules. This might be the case for phospholipids from the inner OM leaflet, as well as for intercalating compounds from the exterior. It could also be expected that structural changes in lipid A affect the steric configuration of further LPS regions, in particular the inner core, which due to phosphates and acidic sugars could be a putative target for cationic agents such as PA $\beta N$.

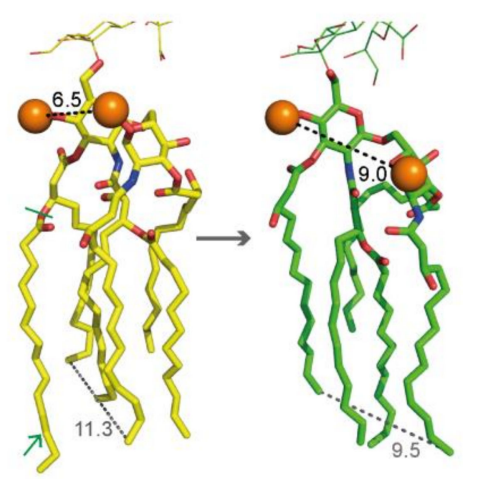

Figure 3. Structures of hexa-acylated and penta-acylated lipid A depicted as yellow and green sticks, respectively (red: oxygen, blue: nitrogen). Phosphates are shown as orange spheres, and distance measurements are shown as dashed lines. The green arrow indicates the myristic acid residue of hexa-acylated lipid A, and the green bar indicates its binding site in lipid A.

\subsection{Further Characterization of Mutant $\Delta l p x M$}

\subsubsection{The Activity of other EPIs}

We also examined whether the drug-sensitizing efficacies of other EPIs were affected by $\operatorname{lp} x M$ deficiency. In contrast to PA $\beta N, N M P$, an arylpiperazine [36], and MBX2319, a pyranopyridine 
compound [37], revealed no or only subtle alteration ( $\leq$ twofold, Table S2) suggesting another mode of action and/or no membrane effects of these compounds.

\subsubsection{The Activity of PMBN}

Polymyxin B nonapeptide (PMBN) is a large cyclic cationic peptide that is known to permeabilize the OM $[1,38]$ by targeting the negatively charged LPS layer [28]. In contrast to PA $\beta N$, the drug-sensitizing activity of PMBN was found to be increased in the $\triangle l p x M$ mutant. With most of the drugs that were tested, a two- to fourfold elevated potentiating efficacy was detected, and the susceptibility to PMBN itself was enhanced by eightfold (Table S2). Hence, different OM-compromising mechanisms of PA $\beta N$ and PMBN appeared likely.

\subsubsection{Intracellular Dye Accumulation}

To explore the functional impact of $l p x M$ deficiency further, we carried out dye accumulation assays. In particular, the effects that could contribute to the decreased drug-sensitizing activity of PA $\beta N$ were of interest. These included: (i) altered drug efflux capacity (ii), decreased intracellular PA $\beta N$ availability, and (iii) the impaired influx of other compounds. Referring to (i), monitoring the Nile red efflux revealed similar functioning of the efflux transporters from the parental strain and mutant $l p x M$ with efflux half-times of $28.3 \mathrm{~s}( \pm 2.4, n=3)$ and $26.3 \mathrm{~s}( \pm 1.9, n=3)$, respectively, whereas that of an efflux-deficient $\triangle a c r B$ mutant was $206.7( \pm 6.2, n=3)$. Notably, PA $\beta N$ did not show any significant alteration in its efflux inhibitory action with this relatively small lipophilic compound (Figure 4a). Concerning (ii), the intracellular accumulation of the PA $\beta N$ degradation product $\beta$-naphthylamine was slightly increased in the lpxM mutant, demonstrating an unimpaired influx of the EPI (Figure 4b). Disproving issue (iii) was that drug susceptibilities were not decreased (Figure 2b, Table S2), and dye uptake was almost unchanged (Figure 4c, Table 1).

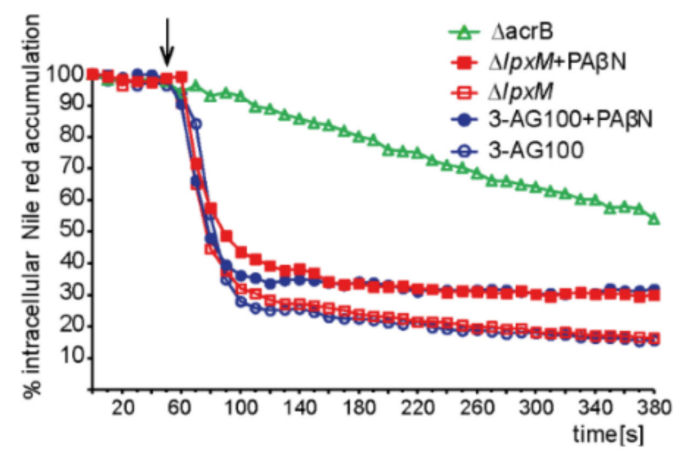

(a)

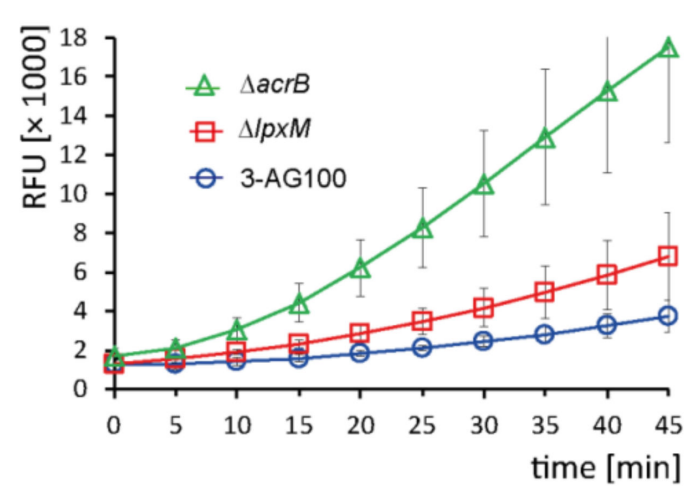

(b)

Figure 4. Cont. 


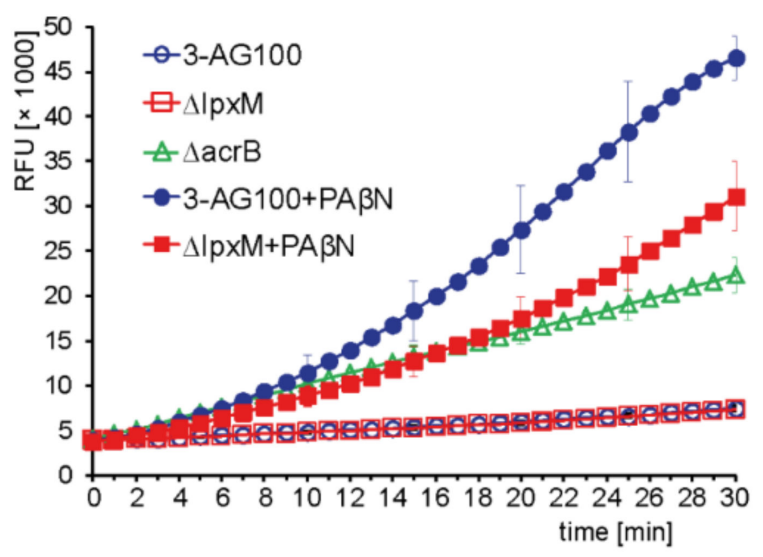

(c)

Figure 4. The kinetics of intracellular dye accumulation in mutant $\Delta l p x M$, parental E. coli 3-AG100, and an efflux-deficient $\triangle a c r B$ derivative from 3-AG100. RFU, relative fluorescence units; error bars indicate \pm standard deviation (SD) from the mean. (a) Real-time efflux of Nile red. The arrow indicates the initiation of efflux by the addition of glucose to de-energized cells; (b) $\beta$-naphthylamine accumulation (degradation product of PA $\beta N$ ). Statistical significance (mutants vs. 3-AG100 at $45 \mathrm{~min}$ ), $p$-values $<0.0001$ $(n=8)$; (c), Resazurin accumulation, determined by measurement of the intracellular degradation product resorufin. Statistical significance (3-AG100 with PA $\beta N$ vs. $\triangle l p x M$ with PA $\beta N)$, $p$-value $<0.0001(n=7)$.

Table 1. Intracellular dye accumulation in the absence and presence of $\mathrm{PA} \beta \mathrm{N}$ and polymyxin $\mathrm{B}$ nonapeptide (PMBN).

\begin{tabular}{|c|c|c|c|c|c|c|c|c|c|}
\hline & \multicolumn{9}{|c|}{$\%$ Dye Accumulation (Relative to $\Delta$ acrB Mutant without Adjuvants) ${ }^{1}$} \\
\hline & \multicolumn{3}{|c|}{ 3-AG100 } & \multicolumn{3}{|c|}{$\Delta l p x M$} & \multicolumn{3}{|c|}{$\Delta a c r B$} \\
\hline Dye & & $+\mathrm{PA} \beta \mathrm{N}$ & + PMBN & & $+\mathrm{PA} \beta \mathrm{N}$ & + PMBN & & $+\mathrm{PA} \beta \mathrm{N}$ & + PMBN \\
\hline Resazurin & $\begin{array}{l}33.4 \\
( \pm 2)\end{array}$ & $\begin{array}{l}208.4 \\
( \pm 11)\end{array}$ & $\begin{array}{l}106.8 \\
( \pm 15)\end{array}$ & $\begin{array}{l}33.1 \\
( \pm 1)\end{array}$ & $\begin{array}{l}139.6 \\
( \pm 18)\end{array}$ & $\begin{array}{l}78.1 \\
( \pm 9)\end{array}$ & $\begin{array}{c}100 \\
( \pm 9)\end{array}$ & $\begin{array}{c}209.7 \\
( \pm 4)\end{array}$ & $\begin{array}{l}226.1 \\
( \pm 4)\end{array}$ \\
\hline Hoechst & $\begin{array}{l}43.1 \\
( \pm 6)\end{array}$ & $\begin{array}{l}44.5 \\
( \pm 7)\end{array}$ & $\begin{array}{l}44.4 \\
( \pm 1)\end{array}$ & $\begin{array}{c}47.2 \\
( \pm 12)\end{array}$ & $\begin{array}{c}47.6 \\
( \pm 11)\end{array}$ & $\begin{array}{l}45.3 \\
( \pm 9)\end{array}$ & $\begin{array}{c}100 \\
( \pm 11)\end{array}$ & $\begin{array}{l}108.6 \\
( \pm 8)\end{array}$ & $\begin{array}{l}108.3 \\
( \pm 3)\end{array}$ \\
\hline Berberine & $\begin{array}{l}31.5 \\
( \pm 7)\end{array}$ & $\begin{array}{l}32.6 \\
( \pm 8)\end{array}$ & $\begin{array}{c}18.7 \\
( \pm 0)\end{array}$ & $\begin{array}{l}22.2 \\
( \pm 1)\end{array}$ & $\begin{array}{l}22.3 \\
( \pm 1)\end{array}$ & $\begin{array}{l}19.6 \\
( \pm 1)\end{array}$ & $\begin{array}{c}100 \\
( \pm 14)\end{array}$ & $\begin{array}{l}117.8 \\
( \pm 14)\end{array}$ & $\begin{array}{l}90.9 \\
( \pm 1)\end{array}$ \\
\hline
\end{tabular}

${ }^{1} \mathrm{PA} \beta \mathrm{N}$ used at $25 \mathrm{mg} / \mathrm{L}$, PMBN used at $10 \mathrm{mg} / \mathrm{L}$. Values determined after $30 \mathrm{~min}$ of accumulation; SD of the mean given in parenthesis. Statistical significance proved for resazurin accumulation in the presence of PA $\beta N$ and of PMBN vs. the absence of adjuvants in all strains, and for berberine accumulation with mutant $\triangle$ acrB (in the presence of PA $\beta \mathrm{N}$ vs. accumulation without adjuvants); $p$-values $<0.01 ; n=7$, assays with PMBN, $n \geq 2$.

Resazurin was the only dye revealing a compromised efficacy of PA $\beta N$ in mutant $\triangle l p x M$ (Figure 4c, Table 1), which is a finding similar to that with large lipophilic drugs. The potency of $\mathrm{PA} \beta \mathrm{N}$ to increase the accumulation of resorufin (intracellular degradation product of resazurin) had previously been shown not only for wild-type E. coli, but even for efflux-deficient strains, suggesting a permeabilizing activity in addition to efflux inhibition [13]. Since the uptake of resazurin, which is a relatively small and more hydrophilic agent, was also significantly enhanced by PMBN (Table 1), an uptake pathway similar to that of large lipophilic drugs appeared likely. Further physicochemical properties, such as the polarity or stiffness of the molecular structure, supposedly determine the influx pathway, too. In contrast to resazurin, Hoechst and berberine accumulation was not increased, neither by PMBN and nor by PA $\beta N$ in E. coli 3-AG100 and mutant $\triangle l p x M$ (Table 1). 


\section{Materials and Methods}

\subsection{Bacterial Strains, Growth Conditions, and Chemicals}

The E. coli strains and mutants that were used and generated in this study are listed in Table 2. Bacteria were grown at $37{ }^{\circ} \mathrm{C}$ overnight using cation-adjusted Müller Hinton (MH) (BBLTM Müller Hinton II, Becton Dickinson, Heidelberg, Germany) or Luria/Miller broth (LB) (Roth, Karlsruhe, Germany) as indicated. Chemicals were purchased from Sigma (Taufkirchen, Germany), NMP was purchased from Chess (Mannheim, Germany), and MBX2139 was a kind gift from Thimothy J. Opperman (Microbiotix, Worcester, MA, USA).

Table 2. Strains and mutants used and engineered in the present study.

\begin{tabular}{|c|c|c|}
\hline E. coli Strains and Mutants & Description & Source \\
\hline 3-AG100 & \multirow{3}{*}{$\begin{array}{l}\text { E. coli K-12 AG100 derivative; overexpression of } a c r B . \\
\text { PA } \beta N-\text { resistant serial selection mutant from 3-AG100. } \\
\text { PA } \beta N \text {-resistant in vitro random mutagenesis mutant from } \\
\text { 3-AG100. }\end{array}$} & Kern et al., 2000 [39] \\
\hline $\mathrm{C} 5 / 1 / 17$ & & Schuster et al., 2014 [21] \\
\hline $\mathrm{CP} 1$ & & This study \\
\hline 3-AG100acrB ${ }_{C P 1}$ & Site-directed mutagenesis mutant from 3-AG100. & This study \\
\hline $\mathrm{CP} 1 a c r B_{\mathrm{WT}}$ & $\mathrm{PA} \beta \mathrm{N}$-resistant site-directed mutagenesis mutant from $\mathrm{CP} 1$. & This study \\
\hline$\Delta l p x M^{1}$ & PA $\beta N$-resistant lpxM knockout mutant from 3-AG100. & This study \\
\hline$\triangle a c r B^{2}$ & Efflux-deficient $a c r B$ knockout mutant from 3-AG100. & Schuster et al., 2014 [21] \\
\hline
\end{tabular}

\subsection{Susceptibility Testing}

The MICs of drugs were determined from freshly grown overnight cultures by a standard twofold broth microdilution assay using 96-well custom plates (Merlin, Bornheim-Hersel, Germany) and inoculums of $5 \times 10^{5} \mathrm{CFU} / \mathrm{mL}$. Assays were performed in the absence and presence of the following adjuvants: PA $\beta N, N M P, M B X 2139$, and PMBN at concentrations of $25 \mathrm{mg} / \mathrm{L}, 100 \mathrm{mg} / \mathrm{L}, 25 \mu \mathrm{M}$, and $10 \mathrm{mg} / \mathrm{L}$, respectively. Approaches with 96-well twofold serial dilutions were also used to determine the MICs of adjuvants and dyes. MICs were determined by visual evaluation in comparison to growth-control wells.

\subsection{In Vitro Random Mutagenesis (Directed Evolution)}

Chromosome-based in vitro random mutagenesis was performed according to a procedure published previously [21]. Briefly, error-prone PCR products were achieved by amplifying acrB with the Mutazym $\mathrm{II}^{\circledR}$ polymerase from the GeneMorph $\mathrm{II}^{\circledR}$, Random Mutagenesis Kit (Stratagene, La Jolla, CA, USA). Subsequently, chromosomal wild-type acrB of E. coli 3-AG100 was substituted by the mutated PCR products using a homologous recombination method (RED/ET ${ }^{\circledR}$ Counter-Selection BAC Modification Kit, Gene Bridges, Heidelberg, Germany). Resulting mutants were subjected to selection with $16 \mathrm{mg} / \mathrm{L}$ CLR in the presence of $25 \mathrm{mg} / \mathrm{L}$ PA $\beta \mathrm{N}$. Screening for stable PA $\beta N$-resistance was done by drug susceptibility assays without and with PA $\beta N$.

\subsection{Sequencing}

Whole genome sequencing was conducted according to a protocol published earlier [40]. Raw sequencing data from mutants C5/1/17 and CP1 and from their parent 3-AG100 were deposited in ENA (European Nucleotide Archive) under sample accession number PRJEB30347. Variants were detected using the CLC Genomics Workbench v 8.0.2 (Qiagen, CLC bio, Aarhus, Denmark) by comparing with the NCBI (National Center for Biotechnology Information) reference sequence (RefSeq) NC_000913.3 from E. coli K-12 substr. MG1655 (accession: PRJNA57779).

Findings were confirmed by Sanger sequencing of purified PCR products (primer given in Table S3) carried out from Microsynth SEQLAB (Göttingen, Germany). 


\subsection{Site-Directed Reconstructions}

Site-directed reconstructions of $a c r B$ from mutant $C P 1$ ( $\left.a c r B_{C P 1}\right)$ within strain 3-AG100 and vice versa of wild-type $a c r B\left(a c r B_{w t}\right)$ within $C P 1$ were performed using the Counter-Selection BAC Modification Kit (Gene Bridges, Heidelberg) according to the manufacturer's instructions. Briefly, $a c r B$ from the parental strain was replaced by an rpsL-neo cassette that was subsequently substituted by the PCR products of the respective $a c r B$ variant. PCR products for homologous recombination procedures were amplified using a proofreading enzyme (Q5 Hot Start High Fidelity DNA Polymerase, New England BioLabs, Frankfurt, Germany), The used oligonucleotides are shown in Table S3.

\subsection{Generation of Knockout Mutants}

The chromosomal lpxM gene knockout mutant was generated using the "Quick \& Easy E. coli Gene Deletion" kit (Gene Bridges, Heidelberg, Germany), as indicated within the manual (oligonucleotides given in Table S3).

\subsection{Intracellular Dye Accumulation Assays}

All of the dye accumulation assays were conducted in the presence of $1 \mathrm{mM}$ of $\mathrm{MgCl}_{2}$, and fluorescence measurements were carried out using the fluorescence plate reader Tecan infinite M200Pro (Männedorf, Switzerland). When assays were carried out in the presence of adjuvants, PA $\beta \mathrm{N}$ and PMBN were added at concentrations of $25 \mathrm{mg} / \mathrm{L}$ and $10 \mathrm{mg} / \mathrm{L}$, respectively.

Nile red efflux assays were performed according to a protocol published earlier [19]. Briefly, cells grown in cation-adjusted $\mathrm{MH}$ were deenergized with $10 \mu \mathrm{M}$ of carbonyl cyanide 3-chlorophenylhydrazone (CCCP) and loaded with Nile red (10 $\mu \mathrm{M}$, two hours). Efflux in washed cells was started by the addition of glucose to a final concentration of $1 \mathrm{mM}$. Fluorescence was measured at excitation and emission wavelengths of $544 \mathrm{~nm}$ and $650 \mathrm{~nm}$, respectively.

The intracellular accumulation of PA $\beta N$ was estimated by fluorescence measurement of the intracellular degradation product $\beta$-naphthylamine. Bacteria from an overnight grown cation-adjusted $\mathrm{MH}$ agar plate were resuspended in phosphate-buffered saline (PBS) with $0.4 \% \mathrm{D}$-glucose to an $\mathrm{OD}_{600}$ of 1 . PA $\beta N$ was added to a final concentration of $200 \mu \mathrm{M}$, and the fluorescence was measured at excitation and emission wavelengths of $320 \mathrm{~nm}$ and $460 \mathrm{~nm}$, respectively.

Resazurin accumulation was determined by measuring the intracellular degradation product resorufin, as described previously [13]

Hoechst 33342 and berberine uptake experiments were carried out as described for PA $\beta \mathrm{N}$ accumulation. Final dye concentrations used were $2.5 \mu \mathrm{M}$ for Hoechst and $30 \mathrm{mg} / \mathrm{L}$ for berberine. The fluorescence of accumulated Hoechst was monitored at excitation and emission wavelengths of $350 \mathrm{~nm}$ and $461 \mathrm{~nm}$, respectively (berberine, $355 \mathrm{~nm}$ and $517 \mathrm{~nm}$ ).

\subsection{LPS Modeling and Visualization}

LPS structures were modeled using the LPS Modeler provided by the CHARMM platform (http:/ / www.charmm-gui.org) [34] with LPS from E. coli K-12, lipid A type 1, and E. coli K-12 lipid A type 3. Visualization and distance measurements were performed using the PyMOL Molecular Graphics System, Version 2.0 Schrödinger, LLC.

\subsection{Statistical Analysis}

SD values were calculated from the mean of $n$ experiments $(n \geq 3$, if not otherwise indicated; $n>3$ if the SD calculated from three replicates was $>10 \%$ ). Statistical significance of differences was analyzed by a two-tailed $t$-tests using the software GraphPad Prism version 7.05 ( $p$-values $<0.05$ represent significance). 


\section{Conclusions}

For the first time, the molecular basis of an OM target of PA $\beta N$ was identified. The study revealed that lpxM deficiency, that was known to cause penta-acylated lipid $\mathrm{A}$, was able to substantially decrease the sensitizing activity of PA $\beta N$ with large lipophilic drugs, which are suggested to use a lipid pathway to access the bacterial cell. Our results provide the basis for further research, particularly with respect to the development of OM permeabilizers, and identify PA $\beta N$ as a perfect template for the design of drug sensitizers with a dual mode of action but as an inadequate tool for the evaluation of efflux phenomena.

Supplementary Materials: The following are available online. Table S1: Susceptibilities of parental E. coli 3-AG100 and of random mutagenesis and reconstructed mutants in the absence and presence of PA $\beta N$ tested in LB medium, Table S2: Susceptibilities of parental E. coli 3-AG100 and of mutant $\Delta l p x M$ in the absence and presence of EPIs and PMBN tested in cation-adjusted MH medium, Table S3: Oligonucleotides used in this study.

Author Contributions: Conceptualization and experiment design, S.S. and J.A.B.; Conducting experiments, S.S. and M.V.; Next generation sequencing, J.W.R.; Data analysis and manuscript writing, S.S.; Supervision, manuscript reviewing, and funding acquisition, W.V.K. All authors read and approved the final manuscript.

Funding: This research was funded in part by the Innovative Medicines Initiative (IMI) Joint Undertaking project no. 115525 ND4BB Translocation (www.translocation.eu, contributions from the European Union's 7th framework program and EFPIA companies).

Acknowledgments: We thank Thimothy J. Opperman from Microbiotix, Worcester, Massachusettes, USA, for kindly providing MBX2139. We also thank Wonpil Im, Presidential Endowed Chair in Health-Science and Engineering, Professor of Biological Sciences and Bioengineering, Lehigh University, Bethlehem, Pennsylvania, USA, for adding the structure of penta-acylated lipid A (lipid A type 3, E. coli K-12 LPS) to the CHARMM-GUI platform.

Conflicts of Interest: The authors declare no conflict of interest.

\section{References}

1. Vaara, M. Agents that increase the permeability of the outer membrane. Microbiol. Rev. 1992, 56, $395-411$. [PubMed]

2. Spengler, G.; Kincses, A.; Gajdacs, M.; Amaral, L. New Roads Leading to Old Destinations: Efflux Pumps as Targets to Reverse Multidrug Resistance in Bacteria. Molecules 2017, 22, 468. [CrossRef] [PubMed]

3. Nikaido, H.; Pages, J.M. Broad-specificity efflux pumps and their role in multidrug resistance of Gram-negative bacteria. FEMS Microbiol. Rev. 2012, 36, 340-363. [CrossRef] [PubMed]

4. Schuster, S.; Vavra, M.; Schweigger, T.M.; Rossen, J.W.A.; Matsumura, Y.; Kern, W.V. Contribution of AcrAB-TolC to multidrug resistance in an Escherichia coli sequence type 131 isolate. Int. J. Antimicrob. Agents 2017, 50, 477-481. [CrossRef] [PubMed]

5. Opperman, T.J.; Nguyen, S.T. Recent advances toward a molecular mechanism of efflux pump inhibition. Front. Microbiol. 2015, 6, 421. [CrossRef] [PubMed]

6. Lomovskaya, O.; Warren, M.S.; Lee, A.; Galazzo, J.; Fronko, R.; Lee, M.; Blais, J.; Cho, D.; Chamberland, S.; Renau, T.; et al. Identification and characterization of inhibitors of multidrug resistance efflux pumps in Pseudomonas aeruginosa: Novel agents for combination therapy. Antimicrob. Agents Chemother. 2001, 45, 105-116. [CrossRef] [PubMed]

7. Laudy, A.E.; Osinska, P.; Namyslowska, A.; Zajac, O.; Tyski, S. Modification of the susceptibility of gram-negative rods producing ES $\beta$ LS to beta-lactams by the efflux phenomenon. PLoS ONE 2015, 10, e0119997. [CrossRef]

8. $\quad$ Pages, J.M.; Lavigne, J.P.; Leflon-Guibout, V.; Marcon, E.; Bert, F.; Noussair, L.; Nicolas-Chanoine, M.H. Efflux pump, the masked side of beta-lactam resistance in Klebsiella pneumoniae clinical isolates. PLoS ONE 2009, 4, e4817. [CrossRef] [PubMed]

9. Laudy, A.E.; Kulinska, E.; Tyski, S. The Impact of Efflux Pump Inhibitors on the Activity of Selected Non-Antibiotic Medicinal Products against Gram-Negative Bacteria. Molecules 2017, 22, 114. [CrossRef] [PubMed]

10. Lamers, R.P.; Cavallari, J.F.; Burrows, L.L. The efflux inhibitor phenylalanine-arginine beta-naphthylamide $(\mathrm{PA} \beta \mathrm{N})$ permeabilizes the outer membrane of gram-negative bacteria. PLoS ONE 2013, 8, e60666. [CrossRef] 
11. Matsumoto, Y.; Hayama, K.; Sakakihara, S.; Nishino, K.; Noji, H.; Iino, R.; Yamaguchi, A. Evaluation of multidrug efflux pump inhibitors by a new method using microfluidic channels. PLoS ONE 2011, 6, e18547. [CrossRef]

12. Misra, R.; Morrison, K.D.; Cho, H.J.; Khuu, T. Importance of Real-Time Assays to Distinguish Multidrug Efflux Pump-Inhibiting and Outer Membrane-Destabilizing Activities in Escherichia coli. J. Bacteriol. 2015, 197, 2479-2488. [CrossRef] [PubMed]

13. Vidal-Aroca, F.; Meng, A.; Minz, T.; Page, M.G.; Dreier, J. Use of resazurin to detect mefloquine as an efflux-pump inhibitor in Pseudomonas aeruginosa and Escherichia coli. J. Microbiol. Methods 2009, 79, 232-237. [CrossRef] [PubMed]

14. Bohnert, J.A.; Schuster, S.; Seeger, M.A.; Fähnrich, E.; Pos, K.M.; Kern, W.V. Site-directed mutagenesis reveals putative substrate binding residues in the Escherichia coli RND efflux pump AcrB. J. Bacteriol. 2008, 190, 8225-8229. [CrossRef]

15. Yu, E.W.; Aires, J.R.; McDermott, G.; Nikaido, H. A periplasmic drug-binding site of the AcrB multidrug efflux pump: A crystallographic and site-directed mutagenesis study. J. Bacteriol. 2005, 187, 6804-6815. [CrossRef] [PubMed]

16. Kinana, A.D.; Vargiu, A.V.; May, T.; Nikaido, H. Aminoacyl $\beta$-naphthylamides as substrates and modulators of AcrB multidrug efflux pump. Proc. Natl. Acad. Sci. USA 2016, 113, 1405-1410. [CrossRef]

17. Vargiu, A.V.; Ruggerone, P.; Opperman, T.J.; Nguyen, S.T.; Nikaido, H. Molecular mechanism of MBX2319 inhibition of Escherichia coli AcrB multidrug efflux pump and comparison with other inhibitors. Antimicrob. Agents Chemother. 2014, 58, 6224-6234. [CrossRef]

18. Nakashima, R.; Sakurai, K.; Yamasaki, S.; Nishino, K.; Yamaguchi, A. Structures of the multidrug exporter AcrB reveal a proximal multisite drug-binding pocket. Nature 2011, 480, 565-569. [CrossRef]

19. Iyer, R.; Ferrari, A.; Rijnbrand, R.; Erwin, A.L. A fluorescent microplate assay quantifies bacterial efflux and demonstrates two distinct compound binding sites in AcrB. Antimicrob. Agents Chemother. 2015, 59, 2388-2397. [CrossRef]

20. Schuster, S.; Vavra, M.; Kern, W.V. Evidence of a Substrate-Discriminating Entrance Channel in the Lower Porter Domain of the Multidrug Resistance Efflux Pump AcrB. Antimicrob. Agents Chemother. 2016, 60, 4315-4323. [CrossRef]

21. Schuster, S.; Kohler, S.; Buck, A.; Dambacher, C.; König, A.; Bohnert, J.A.; Kern, W.V. Random mutagenesis of the multidrug transporter AcrB from Escherichia coli for identification of putative target residues of efflux pump inhibitors. Antimicrob. Agents Chemother. 2014, 58, 6870-6878. [CrossRef] [PubMed]

22. Mao, W.; Warren, M.S.; Black, D.S.; Satou, T.; Murata, T.; Nishino, T.; Gotoh, N.; Lomovskaya, O. On the mechanism of substrate specificity by resistance nodulation division (RND)-type multidrug resistance pumps: The large periplasmic loops of MexD from Pseudomonas aeruginosa are involved in substrate recognition. Mol. Microbiol. 2002, 46, 889-901. [CrossRef]

23. Wehmeier, C.; Schuster, S.; Fahnrich, E.; Kern, W.V.; Bohnert, J.A. Site-directed mutagenesis reveals amino acid residues in the Escherichia coli RND efflux pump AcrB that confer macrolide resistance. Antimicrob. Agents Chemother. 2009, 53, 329-330. [CrossRef]

24. Bohnert, J.A.; Schuster, S.; Fähnrich, E.; Trittler, R.; Kern, W.V. Altered spectrum of multidrug resistance associated with a single point mutation in the Escherichia coli RND-type MDR efflux pump YhiV (MdtF). J. Antimicrob. Chemother. 2007, 59, 1216-1222. [CrossRef] [PubMed]

25. Somerville, J.E., Jr.; Cassiano, L.; Bainbridge, B.; Cunningham, M.D.; Darveau, R.P. A novel Escherichia coli lipid A mutant that produces an antiinflammatory lipopolysaccharide. J. Clin. Investig. 1996, 97, 359-365. [CrossRef] [PubMed]

26. Nikaido, H. Molecular basis of bacterial outer membrane permeability revisited. Microbiol. Mol. Biol. Rev. 2003, 67, 593-656. [CrossRef] [PubMed]

27. Kim, S.; Thiessen, P.A.; Bolton, E.E.; Chen, J.; Fu, G.; Gindulyte, A.; Han, L.; He, J.; He, S.; Shoemaker, B.A.; et al. PubChem Substance and Compound databases. Nucleic Acids Res. 2016, 44, D1202-D1213. [CrossRef]

28. Delcour, A.H. Outer membrane permeability and antibiotic resistance. Biochim. Biophys. Acta 2009, 1794, 808-816. [CrossRef]

29. Karow, M.; Georgopoulos, C. Isolation and characterization of the Escherichia coli msbB gene, a multicopy suppressor of null mutations in the high-temperature requirement gene htrB. J. Bacteriol. 1992, 174, 702-710. [CrossRef] 
30. Clementz, T.; Zhou, Z.; Raetz, C.R. Function of the Escherichia coli msbB gene, a multicopy suppressor of htrB knockouts, in the acylation of lipid A. Acylation by MsbB follows laurate incorporation by HtrB. J. Biol. Chem. 1997, 272, 10353-10360. [CrossRef]

31. Vaara, M.; Nurminen, M. Outer membrane permeability barrier in Escherichia coli mutants that are defective in the late acyltransferases of lipid A biosynthesis. Antimicrob. Agents Chemother. 1999, 43, 1459-1462. [CrossRef] [PubMed]

32. Li, Y.; Wang, Z.; Chen, J.; Ernst, R.K.; Wang, X. Influence of lipid A acylation pattern on membrane permeability and innate immune stimulation. Mar. Drugs 2013, 11, 3197-3208. [CrossRef]

33. Xu, H.; Ling, J.; Gao, Q.; He, H.; Mu, X.; Yan, Z.; Gao, S.; Liu, X. Role of the lpxM lipid A biosynthesis pathway gene in pathogenicity of avian pathogenic Escherichia coli strain E058 in a chicken infection model. Vet. Microbiol. 2013, 166, 516-526. [CrossRef]

34. Jo, S.; Kim, T.; Iyer, V.G.; Im, W. CHARMM-GUI: A web-based graphical user interface for CHARMM. J. Comput. Chem. 2008, 29, 1859-1865. [CrossRef] [PubMed]

35. Ingram, B.O.; Masoudi, A.; Raetz, C.R. Escherichia coli mutants that synthesize dephosphorylated lipid A molecules. Biochemistry 2010, 49, 8325-8337. [CrossRef] [PubMed]

36. Bohnert, J.A.; Kern, W.V. Selected arylpiperazines are capable of reversing multidrug resistance in Escherichia coli overexpressing RND efflux pumps. Antimicrob. Agents Chemother. 2005, 49, 849-852. [CrossRef] [PubMed]

37. Opperman, T.J.; Kwasny, S.M.; Kim, H.S.; Nguyen, S.T.; Houseweart, C.; D’Souza, S.; Walker, G.C.; Peet, N.P.; Nikaido, H.; Bowlin, T.L. Characterization of a Novel Pyranopyridine Inhibitor of the AcrAB Efflux Pump of Escherichia coli. Antimicrob. Agents Chemother. 2014, 58, 722-733. [CrossRef]

38. Hancock, R.E.; Chapple, D.S. Peptide antibiotics. Antimicrob. Agents Chemother. 1999, 43, 1317-1323. [CrossRef] [PubMed]

39. Kern, W.V.; Oethinger, M.; Jellen-Ritter, A.S.; Levy, S.B. Non-target gene mutations in the development of fluoroquinolone resistance in Escherichia coli. Antimicrob. Agents Chemother. 2000, 44, 814-820. [CrossRef]

40. Ferdous, M.; Zhou, K.; Mellmann, A.; Morabito, S.; Croughs, P.D.; de Boer, R.F.; Kooistra-Smid, A.M.; Rossen, J.W.; Friedrich, A.W. Is Shiga Toxin-Negative Escherichia coli O157:H7 Enteropathogenic or Enterohemorrhagic Escherichia coli? Comprehensive Molecular Analysis Using Whole-Genome Sequencing. J. Clin. Microbiol. 2015, 53, 3530-3538. [CrossRef]

Sample Availability: Samples of the compounds are not available from the authors.

(C) 2019 by the authors. Licensee MDPI, Basel, Switzerland. This article is an open access article distributed under the terms and conditions of the Creative Commons Attribution (CC BY) license (http:/ / creativecommons.org/licenses/by/4.0/). 